\title{
Association of Body Fat Percentage With Time in Range Generated by Continuous Glucose Monitoring During Intensive Insulin Pump Therapy in Type 2 Diabetes
}

\section{Yuting Ruan}

nan fang yi ke da xue zhu jiang yi yuan: Zhujiang Hospital

Jiana Zhong

nan fang yi ke da xue zhu jiang yi yuan: Zhujiang Hospital

Rongping Chen

nan fang yi ke da xue zhu jiang yi yuan: Zhujiang Hospital

Zhen Zhang

nan fang yi ke da xue zhu jiang yi yuan: Zhujiang Hospital

Dixing Liu

nan fang yi ke da xue zhu jiang yi yuan: Zhujiang Hospital

Jia Sun

nan fang yi ke da xue zhu jiang yi yuan: Zhujiang Hospital

Hong Chen ( $\nabla$ chenhong123@smu.edu.cn)

nan fang yi ke da xue zhu jiang yi yuan: Zhujiang Hospital https://orcid.org/0000-0002-6317-9955

\section{Research}

Keywords: Time in Range (TIR), Type 2 diabetes, body fat percentage, continuous glucose monitoring, obesity

Posted Date: January 15th, 2021

DOl: https://doi.org/10.21203/rs.3.rs-144260/v1

License: (c) (1) This work is licensed under a Creative Commons Attribution 4.0 International License. Read Full License 


\section{Abstract}

\section{Background}

Obesity is a crucial risk factor associated with type 2 diabetes mellitus (T2DM). Excessive accumulation of body fat may affect the glycemia control in T2DM. This study investigated the relationship between body fat percentage and time in range (TIR) assessed by continuous glucose monitoring (CGM) during short-term intensive insulin pump therapy in T2DM patients.

\section{Method}

A total of 85 T2DM patients were recruited in this cross-sectional study. All participants underwent 72-h CGM period during intensive insulin pump therapy. TIR was defined as the percentage of time spent within the target glucose range of 3.9-10.0 mmol/L. Body composition was measured using bioelectrical impedance analysis (BIA) and overfat was defined as an amount of body fat of at least $25 \%$ of total body mass for men or at least $30 \%$ for women. Multiple linear regression models were used to evaluate the independent association of body fat percentage with TIR after adjusting for confounding factors.

\section{Results}

Compared with normal fat T2DM patients, individual with a higher body fat percentage exhibited lower levels of TIR ( $P=0.004)$ and higher $72 \mathrm{~h}$ mean blood glucose (72h-MBG) $(P=0.001)$ during intensive insulin pump therapy. The presence of overfat inversely correlated with TIR in T2DM populations $(r=-0.306$; $P=0.004)$. Multiple linear regression analysis indicated that body fat percentage was significantly associated with TIR independent of $\mathrm{HbA} 1 \mathrm{c}$, insulin dosage and glycemic variability metrics (All $P$ for trend $<0.05)$. Further adjustment of other body composition parameters, including waist circumference, visceral fat area and muscle quantity, the link between body fat percentage and TIR remained significant (All $P]$ $0.05)$.

\section{Conclusions}

Body fat percentage was significantly associated with TIR in T2DM during intensive insulin pump therapy. Reduction of body fat may be an important therapeutic target to improve glycemic control in obese T2DM patients.

\section{Introduction}

With the rapid economic development, population aging, sedentary lifestyles and excessive eating, the prevalence of both type 2 diabetes mellitus (T2DM) and obesity is rapidly increasing worldwide, reaching pandemic levels. The 2017 International Diabetes Federation (IDF) report showed that the number of diabetes patients in China reached 114.4 million in $2017^{[1]}$. The prevalence of obesity increased from $3.2-10.8 \%$ in adult men and from $6.4-14.9 \%$ in adult women, reaching nearly 90 million in China, making it the country with the largest number of obese people in the world ${ }^{[2]}$. Obesity-related insulin resistance is 
one of the most important pathophysiological mechanisms of T2DM. Compared with normal weight individual, obesity were nearly five times more likely to develop $\mathrm{T}_{2} \mathrm{DM}^{[3]}$, as well as increased the difficulty of glycemia control. Besides, a recent study indicated that high BMI or waist circumference was significantly associated with increased glycemia in non-diabetic population, suggesting poor glycemia control in obese patients ${ }^{[4]}$. Even though BMI or waist circumference is used as an indicator of obesity, they cannot reflect the quantity and distribution of body fat, existing some limitations in assessing body composition. Recently, bioelectrical impedance analysis (BIA) has been developed to measure body composition, which has been shown to be a more convenient, practical, and less invasive method for evaluating body fat ${ }^{[5]}$. Compared with BMI and waist circumference, BIA can obtain body composition indicators such as body fat percentage, muscle mass and visceral fat area. A retrospective crosssectional study showed that both body fat percentage and visceral fat area, rather than BMI, were related to the risk of T2DM, indicating that the accumulation of body fat might closely correlated with the glycemia control ${ }^{[1]}$. Despite the use of body fat percentage in assessing body composition, the relation between body fat percentage and glycemic control in T2DM remains unknown.

Insulin pump therapy as an important method to protect $\beta$-cells and improve glycaemia control, has been widely used in clinical[6]. The 2020 American Diabetes Association (ADA) guidelines recommend that patients using insulin pump therapy should be encouraged to assess glucose levels by continuous glucose monitoring (CGM) ${ }^{[7]}$. CGM continuously captures the glucose profile over a number of days and may be the optimal way to identify an individual's current glycemic status ${ }^{[8]}$. Time in range (TIR) assessed by CGM refers to the time an individual spends within the target glucose range of 3.9$10.0 \mathrm{mmol} / \mathrm{L}$ during a $24 \mathrm{~h}$ period, which provides valuable information about whether the frequency and duration of hypoglycemia or hyperglycemia improve over time. In addition, TIR measurements are useful for evaluating and comparing the response of T2DM to insulin during short-term intensive insulin pump therapy. In recent years, it has been reported that TIR was associated with diabetes complications such as diabetic retinopathy ${ }^{[9]}$, the development of microalbuminuria ${ }^{[10]}$, and peripheral nerve function ${ }^{[11]}$. However, despite increased TIR interest and attention, there is little insight into the relationship between body composition and TIR during short-term intensive insulin pump therapy, which might provide us with more insights for disease management. Therefore, the current study aimed to investigate the association of body fat percentage with TIR obtained from CGM in patients with T2DM during short-term intensive insulin pump therapy.

\section{Research Design And Method}

\section{Study Population}

This cross-sectional observational study was conducted in T2DM patients who had received intensive insulin pump therapy and were monitored by a CGM system at the inpatient department of Zhujiang Hospital of Southern Medical University from July 2019 to October 2020. A total of 85 patients with T2DM according to WHO criteria (1999) ${ }^{[12]}$ were consecutively recruited. The patients, aged $\geq 18$ years, 
had the levels of $\mathrm{HbA1c} \geq 8.0 \%$. Exclusion criteria included type 1 diabetes; pregnancy or lactation; acute diabetic complications, such as diabetic ketoacidosis; use of medications or drugs that may influence glucose metabolism (i.e., steroids and thiazide diuretics); a history of mental disorders, thyroid function disorders, or severe kidney or liver dysfunction. The study protocol was approved by the ethics committees of Zhujiang Hospital of Southern Medical University in accordance with the principles of the Declaration of Helsinki.

\section{Anthropometric and Biochemical Measurements}

Each patient underwent a physical examination that included measurements of body weight, height, waist circumference, hip circumference and systolic blood pressure (SBP), diastolic blood pressure (DBP). The BMI was calculated as weight in kilograms divided by height in meters squared $\left(\mathrm{kg} / \mathrm{m}^{2}\right)$. Waist-to-hip ratio (WHR) is calculated as waist circumference $(\mathrm{cm})$ divided by hip circumference $(\mathrm{cm})$. Blood pressure was measured three times, and the average of three recordings was calculated for further analysis. Patients with a history of hypertension or abnormally high arterial blood pressure (SBP $\geq 140 \mathrm{mmHg}$ or $\mathrm{DBP} \geq 90 \mathrm{mmHg}$ ) were considered as hypertension. Fatty liver disease was evaluated by liver doppler ultrasound examination. Smoking was defined as daily cigarette use for at least 12 months, regardless of the amount.

Venous blood samples were drawn from the antecubital vein after an overnight fast of 8-10 $\mathrm{h}$. The blood analyses were conducted in the laboratory of Zhujiang Hospital, Southern Medical University for the measurement of biochemical markers, such as blood glucose, glycated hemoglobin (HbA1c), C-peptide, lipid profile including triglycerides (TG), total cholesterol (TC), high-density lipoprotein cholesterol (HDL-C) and low-density lipoprotein cholesterol (LDL-C) and uric acid (UA).

\section{Insulin pump therapy}

All participants were treated with short-term intensive insulin therapy using insulin pumps (Paradigm 712 pump, Medtronic Inc., Northridge, CA) with Insulin Aspart (Novo Nordisk, Bagsværd, Denmark) for at least three consecutive days. The initial insulin doses were $0.4-0.5 \mathrm{IU} / \mathrm{kg}$ and total daily doses were divided into $50 \%$ of basal and $50 \%$ of premeal. Dosages were titrated every day based on the fasting and postprandial of three meals capillary blood glucose by an experienced physician in order to achieve glycemic goal. Glycemic targets were defined as fasting/premeal blood glucose less than $6.1 \mathrm{mmol} / \mathrm{L}$ and $2 \mathrm{~h}$ postprandial blood glucose less than $8.0 \mathrm{mmol} / \mathrm{L}$. No other hypoglycemic agents were added during short-term intensive insulin therapy.

\section{CGM Parameters}

All participants were monitored with a CGM system (Abbott Inc., USA) as soon as they receive intensive insulin pump therapy. A CGM system, used for subcutaneous interstitial glucose monitoring, was inserted on the first day and removed after $72 \mathrm{~h}$, generating a total of 288 consecutive sensor value records. After the 3-day monitoring period, the glucose profiles were downloaded from the CGM system, then the TIR and other glycemic metrics were calculated based on the original glucose data recorded by this system. 
TIR was defined as the percentage of time spent within the target glucose range of $3.9-10.0 \mathrm{mmol} / \mathrm{L}$ during a 24-h period, while time above range (TAR) was above the target glucose $(>10.0 \mathrm{mmol} / \mathrm{L})$, time below range (TBR) was below the target glucose $(<3.9 \mathrm{mmol} / \mathrm{L})$. Besides, a series of parameters concerning 72-h mean blood glucose (72-h MBG) and glycemic variability metrics, including standard deviation of glucose (SD), coefficient of variation (CV), mean amplitude of glycemic excursions (MAGE),

and mean of daily differences (MODD) were detected ${ }^{[13]}$. Among them, MAGE was used to evaluate the intra-day glycaemia variability by calculating the arithmetic mean of the differences between consecutive peaks and nadirs, and only excursions of more than one SD of the mean glycemic value were considered $^{[14]}$. MODD, as the average absolute difference of paired sensor glucose values during two successive days, was used to assess the day-to-day glycemic variability ${ }^{[15]}$.

\section{Body composition analysis}

Body composition was measured using Jawon bioelectrical impedance analyzer (BIA) ioi353 (Jawon Medical Co., Ltd., Korea), which processes 15 reactance measurements using tetrapolar 8-point tactile electrode system at three different frequencies $(5,50,250 \mathrm{KHZ})$ at each of five segments of the body (right arm, left arm, right leg, left leg, and trunk), so as to estimate the body fat percentage, muscle quantity and visceral fat area. Before the examination, the participants were required to empty their bladder, wear light clothes, expose their limbs for electrode attachment, and rest for $5 \mathrm{~min}$ to ensure that accurate measurements were captured. The body composition analysis was carried out within 1 week of the intensive insulin pump therapy. This widely used measurement is safe, non-invasive, highly precise with comprehensive indicators and easy to carry out in clinical settings ${ }^{[16,17]}$.

\section{Statistical Analyses}

All statistical analyses were conducted using SPSS 26.0 software (IBM Corporation, Armonk, NY, USA). Normally distributed data are expressed as the mean \pm SD, whereas skewed clinical data are expressed as median value (interquartile range). Categorical variables are expressed as frequencies (percentages). Student's t-test or Mann-Whitney U-tests was used for continuous variables, as appropriate. The chisquare test or Fisher's exact test was carried out for categorical data. The TIRs were divided into three groups with the tertiles as the cut point. The normally distributed multiple samples were assessed using One Way ANOVA, the Post Hoc tests were conducted by using LSD test, S-N-K test or Tamhane T2 test, as appropriate, and Jonckheere-Terpstra test was used for non-normally distributed data. The associations between overfat and TIR was ascertained by Spearman correlation analyses. Multiple linear regression analysis was performed to assess the independent association of body fat percentage with TIR after controlling for covariates including insulin dosage, HbA1c, glycemic variability metrics (i.e., 72-h MBG, SD, MODD) and other body composition parameters, such as waist circumference, BMI, visceral fat area and muscle mass. A value of $P<0.05$ (two-sided) was considered statistically significant.

\section{Results}


The 85 enrolled T2DM patients were grouped into overfat $(n=40)$ and normal fat $(n=45)$ according to the body fat percentage. Overfat was defined as an amount of body fat of at least $25 \%$ of total body mass for men and at least $30 \%$ for women ${ }^{[18,19]}$. All subjects demonstrated high levels of $\mathrm{HbA} 1 \mathrm{c} \geq 8.0 \%$ and all of them had received intensive insulin pump therapy and were monitored by a CGM system. The clinical characteristics of the patients grouped by body fat percentage were summarized in Table 1 . Overall, the mean age of all subjects was $57.6 \pm 10.7$ years, the median duration of diabetes was 66 months, and they had a mean \pm SD HbA1c of $11.0 \pm 1.8 \%$. Patients in overfat group shown a higher level of BMI, waist-to-hip ratio, body fat quantity, and visceral fat area and lower level of muscle/fat ratio (all $P$ for trend $<0.05$ ). Furthermore, TIR, $72 \mathrm{~h}-\mathrm{MBG}$ and $\mathrm{CV}$ also differed statistically across the various groups (all $P<0.05$ ), while the difference could not be observed in $\mathrm{HbA} 1 \mathrm{c}$, diabetes duration or insulin dosage. TIR was $54.3 \pm 17.6 \%$ in the normal fat group and $41.8 \pm 20.8 \%$ in the overfat group $(P=0.004)$. Among the glucose values out of the range of $3.9-10.0 \mathrm{mmol} / \mathrm{L}$, TAR was substantially higher in overfat patients compared with those with normal body fat $(P=0.001)$, while TBR was lower in overfat than that of normal fat ones $(P=0.003)$. Besides, the overfat participants were more likely to suffer from hypertension and fatty liver disease $(P<0.05$ for both). 
Table 1

Characteristics of study participants by body fat percentage.

\begin{tabular}{|lllll|}
\hline Variables & $\begin{array}{l}\text { All subjects } \\
(\mathbf{n = 8 5})\end{array}$ & $\begin{array}{l}\text { Normal fat Group(n } \\
\mathbf{4} \mathbf{4})\end{array}$ & $\begin{array}{l}\text { Overfat Group } \\
(\mathbf{n}=\mathbf{4 0})\end{array}$ & $\begin{array}{l}\text { P } \\
\text { value }\end{array}$ \\
\hline Age(years) & $57.6 \pm 10.7$ & $55.9 \pm 9.8$ & $59.4 \pm 11.4$ & 0.132 \\
\hline Male (n, \%) & $54(63.5 \%)$ & $34(75.6 \%)$ & $20(50.0 \%)$ & 0.018 \\
\hline $\begin{array}{l}\text { Diabetes duration } \\
\text { (months) }\end{array}$ & $\begin{array}{l}66.0(2.3- \\
132.0)\end{array}$ & $60.0(2.3-120.0)$ & $78.0(2.3-$ & 0.602 \\
\hline Insulin doses (IU/kg/d) & $0.50 \pm 0.1$ & $0.49 \pm 0.1$ & $0.52 \pm 0.1$ & 0.307 \\
\hline SBP (mmHg) & $131.5 \pm 18.7$ & $128.5 \pm 19.2$ & $135.0 \pm 17.7$ & 0.112 \\
\hline DBP (mmHg) & $77.2 \pm 10.9$ & $76.5 \pm 9.6$ & $78.0 \pm 12.3$ & 0.528 \\
\hline BMI (kg/m²) & $24.0 \pm 2.9$ & $22.4 \pm 2.2$ & $25.7 \pm 2.7$ & $<0.001$ \\
\hline Waist circumference (cm) & $92.3 \pm 10.9$ & $87.8 \pm 7.7$ & $96.9 \pm 11.8$ & $<0.001$ \\
\hline Hip circumference (cm) & $94.7 \pm 6.5$ & $92.5 \pm 7.0$ & $97.0 \pm 5.2$ & 0.002 \\
\hline Waist-to-hip ratio (WHR) & $0.97 \pm 0.1$ & $0.95 \pm 0.1$ & $1.00 \pm 0.1$ & 0.026 \\
\hline TC (mmol/L) & $5.0(4.2-5.9)$ & $4.8(3.9-5.4)$ & $5.3(4.3-6.1)$ & 0.066 \\
\hline TG (mmol/L) & $1.8(1.2-3.0)$ & $1.5(1.1-3.0)$ & $1.9(1.3-3.2)$ & 0.297 \\
\hline HDL-C (mmol/L) & $1.0 \pm 0.2$ & $1.0 \pm 0.3$ & $1.0 \pm 0.2$ & 0.990 \\
\hline LDL-C (mmol/L) & $3.1 \pm 1.1$ & $3.0 \pm 1.0$ & $3.3 \pm 1.2$ & 0.191 \\
\hline HbA1c (\%) & $11.0 \pm 1.8$ & $10.8 \pm 1.8$ & $11.2 \pm 1.7$ & 0.305 \\
\hline Fasting C-peptide (ng/mL) & $1.6(1.2-2.8)$ & $1.4(1.1-2.3)$ & $2.0(1.2-3.3)$ & 0.101 \\
\hline $\begin{array}{l}\text { Postprandial C-peptide } \\
\text { (ng/mL) }\end{array}$ & $2.8(1.9-4.7)$ & $2.6(1.8-4.5)$ & $2.9(2.2-5.3)$ & 0.127 \\
\hline Uric acid (umol/L) & $324.0(266.5-$ & $332.0(266.5-393.5)$ & $267.3(321.0-$ & 0.819 \\
\hline 72 h-MBG (mmol/L) & $10.4 \pm 2.3$ & $9.6 \pm 2.1$ & $415.8)$ & 0.001 \\
\hline & & & $11.3 \pm 2.2$ & \\
\hline
\end{tabular}

Data are represented as mean $\pm S D$, median [25th to 75th percentile range], or number (\%). DBP, diastolic blood pressure; SBP, systolic blood pressure; BMI, body mass index; TC, total cholesterol; TG, Triglycerides; HDL-C, high density lipoprotein cholesterol; LDL-C, low density lipoprotein cholesterol; 72 h-MBG, 72-h mean blood glucose concentration; SDBG, standard deviation of blood glucose; CV, coefficient of variation; MAGE, mean amplitude of glycemic excursions; MODD, mean of daily differences; TIR, time in range; TAR, time above range; TBR, time below range; Muscle/fat ratio: muscle quantity/body fat quantity. 


\begin{tabular}{|c|c|c|c|c|}
\hline Variables & $\begin{array}{l}\text { All subjects } \\
(n=85)\end{array}$ & $\begin{array}{l}\text { Normal fat Group(n } \\
=45)\end{array}$ & $\begin{array}{l}\text { Overfat Group } \\
(n=40)\end{array}$ & $\begin{array}{l}P \\
\text { value }\end{array}$ \\
\hline SDBG (mmol/L) & $3.3(2.8-3.9)$ & $3.3(2.7-3.8)$ & $3.4(2.8-4.1)$ & 0.489 \\
\hline CV (\%) & $34.2 \pm 7.7$ & $35.9 \pm 7.6$ & $32.3 \pm 7.6$ & 0.033 \\
\hline MAGE (mmol/L) & $4.6 \pm 1.3$ & $4.4 \pm 1.2$ & $4.8 \pm 1.3$ & 0.139 \\
\hline MODD (mmol/L) & $3.3(2.4-3.9)$ & $3.0(2.2-3.8)$ & $3.5(2.5-4.2)$ & 0.109 \\
\hline TIR (\%) & $48.4 \pm 20.1$ & $54.3 \pm 17.6$ & $41.8 \pm 20.8$ & 0.004 \\
\hline TAR (\%) & $49.0 \pm 22.5$ & $41.7 \pm 20.9$ & $57.4 \pm 21.5$ & 0.001 \\
\hline TBR (\%) & $0(0-1.7)$ & $0.4(0-8.0)$ & $0(0-0.9)$ & 0.003 \\
\hline Muscle quantity (kg) & $44.0 \pm 8.0$ & $44.9 \pm 7.5$ & $43.1 \pm 8.4$ & 0.305 \\
\hline Body fat quantity (kg) & $16.7 \pm 5.0$ & $13.5 \pm 3.6$ & $20.3 \pm 3.6$ & $<0.001$ \\
\hline Muscle/fat ratio & $2.7(2.1-3.2)$ & $3.2(2.8-3.8)$ & $2.1(1.9-2.5)$ & $<0.001$ \\
\hline Body fat percentage & $25.8 \pm 6.3$ & $21.8 \pm 5.1$ & $30.4 \pm 4.0$ & $<0.001$ \\
\hline Visceral fat area $\left(\mathrm{cm}^{2}\right)$ & $101.8 \pm 37.0$ & $88.0 \pm 30.8$ & $117.7 \pm 37.6$ & $<0.001$ \\
\hline Hypertension(n, \%) & $38(44.7 \%)$ & $13(28.9 \%)$ & $25(62.5 \%)$ & 0.002 \\
\hline Fatty liver(n, \%) & $55(64.7 \%)$ & $24(55.8 \%)$ & $31(83.8 \%)$ & 0.007 \\
\hline Current smoker(n, \%) & $22(25.9 \%)$ & $15(34.1 \%)$ & $7(17.5 \%)$ & 0.084 \\
\hline \multicolumn{5}{|c|}{$\begin{array}{l}\text { Data are represented as mean } \pm \text { SD, median [25th to } 75 \text { th percentile range], or number (\%). DBP, } \\
\text { diastolic blood pressure; SBP, systolic blood pressure; BMI, body mass index; TC, total cholesterol; TG, } \\
\text { Triglycerides; HDL-C, high density lipoprotein cholesterol; LDL-C, low density lipoprotein cholesterol; } \\
72 \text { h-MBG, 72-h mean blood glucose concentration; SDBG, standard deviation of blood glucose; CV, } \\
\text { coefficient of variation; MAGE, mean amplitude of glycemic excursions; MODD, mean of daily } \\
\text { differences; TIR, time in range; TAR, time above range; TBR, time below range; Muscle/fat ratio: } \\
\text { muscle quantity/body fat quantity. }\end{array}$} \\
\hline
\end{tabular}

Next, all of the participants were stratified into groups according to tertiles of the TIR (Tertile 1 [T1]: $\leq 43.75 \%$; Tertile 2 [T2]: 43.76-57.29\%; Tertile 3 [T3]: $\geq 57.30 \%$ ). Table 2 depicts the characteristics of patients by TIR tertiles. Significant differences were detected in TIR and glycemic variability measures including 72-h MBG, SDBG, CV, and MODD among the TIR tertiles (all $P$ for trend $<0.05$ ). Whereas the body composition indicators, such as BMl, waist circumference, and body fat percentage, did not reach statistical differences between groups. Participants with the highest tertiles of TIR (T3) exhibited lower levels of triglycerides $(P=0.008)$. Considering the cut-off value of body fat percentage is different in gender, we thus further compared the proportion of overfat patients defined by body fat percentage 
stratified by sex in different TIR tertiles groups. In general, with the ascending tertiles of TIR, the prevalence of overfat assessed by body fat percentage decreased $(P=0.041)$ (Fig. 1A). However, there were no such trends in the categories of BMI, waist circumference and visceral fat area (all $P>0.05$ ), suggesting body fat percentage may be a strong predictor of glycemic control in T2DM patients during insulin pump therapy (Fig. 1B-D). 
Table 2

Characteristics of patients according to the TIR tertiles.

\section{TIR tertiles}

\begin{tabular}{|c|c|c|c|c|}
\hline Variables & $\begin{array}{l}\mathrm{T} 1(\leq 43.75 \%) \\
(\mathrm{n}=29)\end{array}$ & $\begin{array}{l}\text { T2 (43.76- } \\
57.29 \%) \\
(n=29)\end{array}$ & $\begin{array}{l}\text { T3 ( } \geq 57.30 \%) \\
(n=27)\end{array}$ & $\begin{array}{l}P \\
\text { value }\end{array}$ \\
\hline Age(years) & $58.6 \pm 10.1$ & $55.5 \pm 11.7$ & $58.7 \pm 10.2$ & 0.428 \\
\hline Male (n, \%) & $18(62.1 \%)$ & $20(69.0 \%)$ & $16(59.3 \%)$ & 0.737 \\
\hline Diabetes duration (months) & $96.0(4.0-126.0)$ & $54.0(1.3-141.0)$ & $60.0(3.0-180.0)$ & 0.714 \\
\hline Insulin dosage (IU/kg/d) & $0.55 \pm 0.1$ & $0.50 \pm 0.1$ & $0.45 \pm 0.1$ & 0.006 \\
\hline SBP $(m m H g)$ & $134.2 \pm 18.1$ & $131.2 \pm 19.7$ & $129.0 \pm 18.6$ & 0.578 \\
\hline DBP $(\mathrm{mmHg})$ & $76.7 \pm 10.3$ & $79.2 \pm 10.5$ & $75.6 \pm 12.0$ & 0.453 \\
\hline $\mathrm{BMI}\left(\mathrm{kg} / \mathrm{m}^{2}\right)$ & $24.0 \pm 2.4$ & $23.8 \pm 3.7$ & $24.1 \pm 2.6$ & 0.949 \\
\hline Waist circumference $(\mathrm{cm})$ & $94.3 \pm 12.6$ & $91.1 \pm 10.6$ & $91.4 \pm 9.1$ & 0.493 \\
\hline Hip circumference $(\mathrm{cm})$ & $94.5 \pm 4.5$ & $94.0 \pm 9.0$ & $95.7 \pm 5.4$ & 0.591 \\
\hline Waist-to-hip ratio (WHR) & $1.00 \pm 0.1$ & $0.97 \pm 0.1$ & $0.95 \pm 0.1$ & 0.237 \\
\hline $\mathrm{TC}(\mathrm{mmol} / \mathrm{L})$ & $5.1(4.4-6.2)$ & $5.0(4.3-5.9)$ & $4.6(3.8-5.6)$ & 0.058 \\
\hline TG (mmol/L) & $2.1(1.3-3.4)$ & $2.1(1.3-3.8)$ & $1.2(0.8-2.0)$ & 0.008 \\
\hline $\mathrm{HDL}-\mathrm{C}(\mathrm{mmol} / \mathrm{L})$ & $1.0 \pm 0.2$ & $1.0 \pm 0.3$ & $1.0 \pm 0.3$ & 0.374 \\
\hline LDL-C (mmol/L) & $3.3 \pm 1.2$ & $3.1 \pm 1.0$ & $2.9 \pm 1.1$ & 0.386 \\
\hline HbA1c (\%) & $11.6 \pm 1.7$ & $10.9 \pm 1.2$ & $10.6 \pm 2.2$ & 0.080 \\
\hline Fasting C-peptide (ng/mL) & $1.9(1.2-3.0)$ & $1.4(0.9-2.2)$ & $1.5(1.1-3.1)$ & 0.495 \\
\hline $\begin{array}{l}\text { Postprandial C-peptide } \\
(\mathrm{ng} / \mathrm{mL})\end{array}$ & $2.8(2.2-4.9)$ & $2.7(1.6-5.0)$ & $3.5(1.9-4.7)$ & 0.994 \\
\hline Uric acid (umol/L) & $\begin{array}{l}321.0(240.5- \\
410.5)\end{array}$ & $\begin{array}{l}332.0(251.5- \\
389.5)\end{array}$ & $\begin{array}{l}317.0(277.0- \\
409.0)\end{array}$ & 0.920 \\
\hline TIR (\%) & $26.6 \pm 13.1$ & $49.8 \pm 3.7$ & $70.4 \pm 8.3$ & $<0.001$ \\
\hline
\end{tabular}

Data are represented as mean $\pm S D$, median [25th to 75th percentile range], or number (\%). DBP, diastolic blood pressure; SBP, systolic blood pressure; BMI, body mass index; TC, total cholesterol; TG, Triglycerides; HDL-C, high density lipoprotein cholesterol; LDL-C, low density lipoprotein cholesterol; $72 \mathrm{~h}-\mathrm{MBG}, 72$-h mean blood glucose concentration; SDBG, standard deviation of blood glucose; CV, coefficient of variation; MAGE, mean amplitude of glycemic excursions; MODD, mean of daily differences; TIR, time in range; Muscle/fat ratio: muscle quantity/body fat quantity. 


\begin{tabular}{|c|c|c|c|c|}
\hline \multicolumn{4}{|c|}{ TIR tertiles } & \multirow[b]{2}{*}{$<0.001$} \\
\hline 72 h-MBG (mmol/L) & $12.8 \pm 1.5$ & $10.2 \pm 1.0$ & $8.1 \pm 1.1$ & \\
\hline SDBG (mmol/L) & $3.8(3.2-4.8)$ & $3.5(3.1-4.0)$ & $2.7(2.5-3.4)$ & $<0.001$ \\
\hline CV (\%) & $30.9 \pm 7.5$ & $36.2 \pm 8.5$ & $35.7 \pm 5.9$ & 0.014 \\
\hline MAGE (mmol/L) & $4.9 \pm 1.3$ & $4.5 \pm 1.4$ & $4.3 \pm 1.1$ & 0.178 \\
\hline MODD (mmol/L) & $3.5(2.7-4.1)$ & $3.5(2.4-4.6)$ & $3.0(1.9-3.4)$ & 0.017 \\
\hline Muscle quantity (kg) & $43.2 \pm 8.4$ & $44.6 \pm 9.0$ & $44.4 \pm 6.4$ & 0.772 \\
\hline Body fat quantity $(\mathrm{kg})$ & $17.5 \pm 3.1$ & $16.1 \pm 6.9$ & $16.6 \pm 4.1$ & 0.498 \\
\hline Muscle/fat ratio & $2.5(2.0-2.9)$ & $2.7(2.3-3.3)$ & $2.8(2.1-3.7)$ & 0.240 \\
\hline Body fat percentage & $27.5 \pm 5.4$ & $24.3 \pm 7.3$ & $25.7 \pm 5.8$ & 0.149 \\
\hline Visceral fat area $\left(\mathrm{cm}^{2}\right)$ & $111.8 \pm 33.3$ & $96.4 \pm 44.7$ & $96.8 \pm 30.2$ & 0.180 \\
\hline Hypertension(n, \%) & $15(51.7 \%)$ & $12(41.4 \%)$ & $11(40.7 \%)$ & 0.644 \\
\hline Fatty liver(n, \%) & $20(74.1 \%)$ & $18(66.7 \%)$ & $17(65.4 \%)$ & 0.760 \\
\hline Current smoker(n, \%) & $7(25.0 \%)$ & $7(24.1 \%)$ & $8(29.6 \%)$ & 0.883 \\
\hline \multicolumn{5}{|c|}{$\begin{array}{l}\text { Data are represented as mean } \pm \text { SD, median [25th to 75th percentile range], or number (\%). DBP, } \\
\text { diastolic blood pressure; SBP, systolic blood pressure; BMI, body mass index; TC, total cholesterol; TG, } \\
\text { Triglycerides; HDL-C, high density lipoprotein cholesterol; LDL-C, low density lipoprotein cholesterol; } \\
72 \text { h-MBG, 72-h mean blood glucose concentration; SDBG, standard deviation of blood glucose; CV, } \\
\text { coefficient of variation; MAGE, mean amplitude of glycemic excursions; MODD, mean of daily } \\
\text { differences; TIR, time in range; Muscle/fat ratio: muscle quantity/body fat quantity. }\end{array}$} \\
\hline
\end{tabular}

The correlation between overfat and TIR were performed with Spearman's analysis, the data revealed that the presence of overfat negatively correlated with $\operatorname{TIR}(r=-0.306 ; P=0.004)$. Additionally, in a multiple linear regression model, strong relationship existed between body fat percentage and TIR after adjusting for HbA1c and other glycemic variability metrics, including 72-h MBG, SD and MODD (Model 1, $\beta=0.114$, $P=0.008)$. Furthermore, we found the insulin dose was lower in the higher TIR tertile group during intensive insulin pump therapy $(P=0.006$, Table 2$)$. After adjusting insulin dosage based on Model 1 , strong link was remain observed between body fat percentage and TIR (Model 2, $\beta=0.106, P=0.014$ ). Moreover, the association persisted when considering other confounders, including waist circumference, visceral fat area and muscle quantity (All $P<0.05$ ). However, the link between body fat percentage and TIR was weakened after adjusting BMI $(P=0.074)$ (Table 3$)$. 
Table 3

Association of body fat percentage with TIR by linear regression analyses after adjusting for confounding factors.

\begin{tabular}{|c|c|c|c|}
\hline Models & SE & Standardized coefficients B & $P$ value \\
\hline \multicolumn{4}{|l|}{ Model 1} \\
\hline body fat percentage & 0.001 & 0.114 & 0.008 \\
\hline \multicolumn{4}{|l|}{ Model 2} \\
\hline body fat percentage & 0.001 & 0.106 & 0.014 \\
\hline insulin dosage & 0.078 & 0.056 & 0.250 \\
\hline \multicolumn{4}{|l|}{ Model 3} \\
\hline body fat percentage & 0.001 & 0.092 & 0.030 \\
\hline waist circumference & 0.001 & -0.055 & 0.200 \\
\hline \multicolumn{4}{|l|}{ Model 4} \\
\hline body fat percentage & 0.002 & 0.094 & 0.074 \\
\hline BMI & 0.004 & 0.020 & 0.705 \\
\hline \multicolumn{4}{|l|}{ Model 5} \\
\hline body fat percentage & 0.001 & 0.098 & 0.030 \\
\hline visceral fat area & 0.000 & 0.032 & 0.466 \\
\hline \multicolumn{4}{|l|}{ Model 6} \\
\hline body fat percentage & 0.001 & 0.117 & 0.012 \\
\hline muscle quantity & 0.001 & 0.036 & 0.489 \\
\hline
\end{tabular}

Model 1 was adjusted for 72-h MBG, SDBG, MODD and HbA1c.

Model 2 includes all variables in model 1 plus insulin dosage.

Model 3 includes all variables in model 2 plus waist circumference.

Model 4 includes all variables in model 2 plus BMI.

Model 5 includes all variables in model 2 plus visceral fat area.

Model 6 includes all variables in model 2 plus muscle quantity.

TIR, time in range; 72-h MBG, 72-h mean blood glucose concentration; SDBG, standard deviation of blood glucose; MODD, mean of daily differences.

\section{Discussion}


Obesity is known to have an insidious onset and predisposes toward several metabolic disturbances that threaten human health. Excessive ectopic accumulation of adipose tissue in the body and changes in body composition are crucial factors in the development of obesity-related insulin resistance and $\mathrm{T}_{2} \mathrm{DM}^{[20]}$, as well as affect the glycemic control. A retrospective cohort study indicated that baseline BMI is one of the most accurate predictors of the future glycemic control in T2DM patients ${ }^{[21]}$. Previous crosssectional study has also showed that $\mathrm{HbA} 1 \mathrm{c}$ was significant and positive associated with increased waist circumference in T2DM participants ${ }^{[22]}$. However, the parameter of BMI or waist circumference to assess obesity-related complications is not enough. In this study, we elaborated on the correlation between body composition, assessed by bioelectrical impedance analyzer (BIA), and TIR assessed by CGM in T2DM patients during short-term intensive insulin pump therapy. Our findings showed that body composition, particularly high body fat percentage may contribute to decreased TIR in obese T2DM population. To the best of our knowledge, this was the first study to evaluate the association between body composition and CGM-assessed TIR especially during short-term intensive insulin pump therapy in Chinese obese T2DM patients.

As a convenient, practical and less invasive method to assess body composition, BIA was widely used in clinical practice. A cross-sectional study has demonstrated that total body fat mass assessed by BIA were strongly associated with insulin resistance in T2DM ${ }^{[23]}$. Besides, Hameed EK et al. studied the impact of visceral fat in T2DM and found that visceral adiposity index was positively associated with the presence of T2DM and had a significant negative outcome over glycemic control ${ }^{[24,25]}$. Since ectopic fat accumulation was significantly related to body fat percentage measured by BIA in a previous study ${ }^{[26]}$, it is possible that body fat percentage can reflect ectopic fat quantity and may become an optimal predictor of T2DM glycemia management. Currently, the results of our study showed that body fat percentage was significantly and independently correlated with glycemia control. We also found a poor controlled glycemia in T2DM patients with relatively high body fat percentage during intensive hypoglycemic therapy, suggesting the accumulation of body fat may be the main cause of the poor glycemia control in obese T2DM populations.

Intensive insulin pump therapy has been of value in T2DM who fail to achieve optimal glycemic control ${ }^{[27]}$. More physiological delivery of insulin by the pump has been proven for reduction of glucose toxicity, resulting in improvement of insulin resistance in obese T2DM patients ${ }^{[28]}$. With advances in CGM technology, time in range (TIR) of $3.9-10 \mathrm{mmol} / \mathrm{L}$ has been introduced by the 2020 ADA guidelines as an intuitive and key parameter of short-term glycemic management ${ }^{[7]}$. A series of previous studies have also shown that TIR not only could be used to assess the risk of microvascular complications ${ }^{[9,11,29]}$, but also predict the all-cause mortality from cardiovascular events in T2 $\mathrm{DM}^{[30]}$, further supporting TIR as an acceptable glucose metric as well as a reasonable end point for clinical trials. In the current study, with ascending tertiles of TIR, the percentage of overfat patients classified by body fat percentage decreased as compared to other grouping methods (such as BMI, waist circumference or visceral fat area), suggesting body fat percentage may had a more significant negative outcome over glycemic control during intensive insulin pump therapy. Recently, a study demonstrated that regardless of mean glucose, 
$\mathrm{HbA1c}$ or glycemic variability metrics had an impact on $\operatorname{TIR}^{[31,32]}$. Based on the previous study, we further found a robust correlation between body fat percentage and TIR even after adjusting for these above factors. Besides, the effect of body fat percentage on TIR was also independent of other body composition parameters, including waist circumference, visceral fat area and muscle quantity. However, it is notable that the adjustment for BMI, to some extent attenuated the association of body fat percentage with TIR. A possible explanation could be a significant correlation between body fat percentage and BMI in our study samples $(r=0.492 ; P<0.001$; data not shown), resulting in multicollinearity in the linear regression model and thereby affect the result. Nevertheless, our study provides evidence of an independent effect of body fat percentage on TIR.

Furthermore, glycemic variability was also taken into consideration in our study when evaluating quality of glycemic control during insulin pump therapy in T2DM. A previous cross-sectional study reported that subjects with a higher BMI or waist circumference had higher levels of 72-h MBG assessed by CGM system ${ }^{[4]}$. Our study was in consistent with the result of previous study, demonstrating a higher levels of 72-h MBG in obese T2DM patients than that of non-obese ones during insulin pump therapy. However, the decreased of glycemic variability CV was observed in patients with high body fat percentage. Among glycemic variability parameters, CV was significantly correlated with the risk of hypoglycemia ${ }^{[33]}$. In the current study, TBR, defined as the percentage of time spent below the target glucose range $(\varangle 3.9 \mathrm{mmol} / \mathrm{L})$, was lower in participants with higher body fat percentage, indicating the risk of hypoglycemic was relatively decreased during intensive insulin pump therapy in obese subjects. A previous study reported that obese patients exhibited a little bit better pancreatic $\beta$-cell function in comparison with that observed in the non-obese subjects ${ }^{[34]}$, which may contribute to decreased glucose fluctuation in T2DM individual with relatively high body fat percentage.

Several limitations of this study should be addressed. First, this was a cross-sectional study, and thus we could not examine the causal relationship between body composition and TIR. In addition, the measurement of TIR for a 72-h period may not represent the glycemic control of the participants during the whole period of intensive insulin pump therapy. Besides, considering the small overall sample size in our study, the results be replicated in larger study populations is warranted. Finally, we estimated body composition based on the BIA, not by the "gold standard" method, such as computer tomography (CT) and Magnetic resonance imaging (MRI); however, CT or MRI is expensive and not easily feasible in a relatively large-scale study, and we believe that proxy measures are reliable according to the previous studies $^{[35]}$.

\section{Conclusions}

High body fat percentage adversely affect the glycemic control in T2DM individual during intensive insulin pump therapy. Our findings suggested that quantitative assessment of body fat and further consider body fat percentage as part of management strategy are fundamentally important in clinical 
practice. Reduction of body fat may be crucial therapeutic target in improving glycemic control in obese T2DM patients.

\section{List Of Abbreviations}

\begin{tabular}{|ll|}
\hline T2DM & type 2 diabetes mellitus \\
\hline BMI & Body mass index \\
\hline BIA & bioelectrical impedance analysis \\
TIR & Continuous Glucose Monitoring \\
TAR & time in range \\
TBR & time above range \\
\hline 72h-MBG & 72-h mean blood glucose \\
\hline SDBG & standard deviation of blood glucose \\
CV & coefficient of variation \\
MAGE & mean amplitude of glycemic excursions \\
MODD & mean of daily differences \\
\hline
\end{tabular}

\section{Declarations}

\section{Ethics approval and consent to participate}

The study was approved by the ethics committees of Zhujiang Hospital of Southern Medical University in accordance with the principles of the Declaration of Helsinki.

\section{Consent for publication}

Written informed consent for publication was obtained from all participants.

\section{Availability of data and materials}

The datasets used and analyzed during the current study are available from the corresponding author on reasonable request.

\section{Competing interests}

The authors declare that they have no competing interests.

\section{Funding}


This work was supported by grants to Yuting Ruan (the Medical Science and Technology Research Foundation of Guangdong Province of China no. 20181031141744728, the Guangdong Basic and Applied Basic Research Fund Project no. 2019A1515110658) and Hong Chen (the National Natural Science Foundation of China no. 81770835, the Key-Area Research and Development Program of Guangdong Province no. 2019B020230001, and the Guangzhou Science and Technology Program Project, China no. 201803040012).

\section{Authors' contributions}

All of the listed authors contributed to this study. YTR and JNZ analyzed, interpreted data and were major contributors in writing the manuscript. They contributed equally to this work and were considered to be co-first authors. RPC collected clinical data of patients. ZZ and DXL participated in interpretation of data. JS and $\mathrm{HC}$ designed this research and substantively revised it. All authors read and approved the final manuscript. And we have agreed both to be personally accountable for the author's own contributions and to be accountable for all aspects of the work in ensuring that questions related to the accuracy or integrity of any part of the work are appropriately investigated and resolved.

\section{Acknowledgements}

Not applicable.

\section{References}

1. Chen $Y, H e D$, Yang $T$, et al. Relationship between body composition indicators and risk of type 2 diabetes mellitus in Chinese adults. BMC public health 2020, 20(1): 452.

2. Blüher M. Obesity: global epidemiology and pathogenesis. Nat Rev Endocrinol 2019, 15(5): 288-298.

3. Wilding JP. The importance of weight management in type 2 diabetes mellitus. International journal of clinical practice 2014, 68(6): 682-691.

4. Noordam R, Huurman NC, Wijsman CA, et al. High Adiposity Is Associated With Higher Nocturnal and Diurnal Glycaemia, but Not With Glycemic Variability in Older Individuals Without Diabetes. Front Endocrinol (Lausanne) 2018, 9: 238.

5. Dulloo AG, Jacquet J, Solinas G, Montani JP, Schutz Y. Body composition phenotypes in pathways to obesity and the metabolic syndrome. International journal of obesity (2005) 2010, 34 Suppl 2: S4-17.

6. Weng J, Li Y, Xu W, et al. Effect of intensive insulin therapy on beta-cell function and glycaemic control in patients with newly diagnosed type 2 diabetes: a multicentre randomised parallel-group trial. Lancet (London, England) 2008, 371(9626): 1753-1760.

7. Diabetes Technology: Standards of Medical Care in Diabetes-2020. Diabetes care 2020, 43(Suppl 1): S77-s88.

8. Battelino T, Danne T, Bergenstal RM, et al. Clinical Targets for Continuous Glucose Monitoring Data Interpretation: Recommendations From the International Consensus on Time in Range. Diabetes care 
2019, 42(8): 1593-1603.

9. Lu J, Ma X, Zhou J, et al. Association of Time in Range, as Assessed by Continuous Glucose Monitoring, With Diabetic Retinopathy in Type 2 Diabetes. Diabetes care 2018, 41(11): 2370-2376.

10. Beck RW, Bergenstal RM, Riddlesworth TD, et al. Validation of Time in Range as an Outcome Measure for Diabetes Clinical Trials. Diabetes care 2019, 42(3): 400-405.

11. Li F, Zhang Y, Li H, et al. TIR generated by continuous glucose monitoring is associated with peripheral nerve function in type 2 diabetes. Diabetes research and clinical practice 2020, 166 : 108289.

12. Alberti KG, Zimmet PZ. Definition, diagnosis and classification of diabetes mellitus and its complications. Part 1: diagnosis and classification of diabetes mellitus provisional report of a WHO consultation. Diabet Med 1998, 15(7): 539-553.

13. Hu YM, Zhao LH, Zhang XL, et al. Association of glycaemic variability evaluated by continuous glucose monitoring with diabetic peripheral neuropathy in type 2 diabetic patients. Endocrine 2018, 60(2): 292-300.

14. Service FJ. Glucose variability. Diabetes 2013, 62(5): 1398-1404.

15. Ohara M, Fukui T, Ouchi $M$, et al. Relationship between daily and day-to-day glycemic variability and increased oxidative stress in type 2 diabetes. Diabetes research and clinical practice 2016, 122: 6270.

16. Thomas E, Gupta PP, Fonarow GC, Horwich TB. Bioelectrical impedance analysis of body composition and survival in patients with heart failure. Clinical cardiology 2019, 42(1): 129-135.

17. Steinberg A, Manlhiot C, Li P, et al. Development and Validation of Bioelectrical Impedance Analysis Equations in Adolescents with Severe Obesity. The Journal of nutrition 2019, 149(7): 1288-1293.

18. Frankenfield DC, Rowe WA, Cooney RN, Smith JS, Becker D. Limits of body mass index to detect obesity and predict body composition. Nutrition (Burbank, Los Angeles County, Calif) 2001, 17(1): 2630 .

19. Ortega FB, Lee DC, Katzmarzyk PT, et al. The intriguing metabolically healthy but obese phenotype: cardiovascular prognosis and role of fitness. European heart journal 2013, 34(5): 389-397.

20. Unger RH, Clark GO, Scherer PE, Orci L. Lipid homeostasis, lipotoxicity and the metabolic syndrome. Biochimica et biophysica acta 2010, 1801(3): 209-214.

21. Hertroijs DFL, Elissen AMJ, Brouwers $M$, et al. A risk score including body mass index, glycated haemoglobin and triglycerides predicts future glycaemic control in people with type 2 diabetes. Diabetes, obesity \& metabolism 2018, 20(3): 681-688.

22. Fasil A, Biadgo B, Abebe M. Glycemic control and diabetes complications among diabetes mellitus patients attending at University of Gondar Hospital, Northwest Ethiopia. Diabetes, metabolic syndrome and obesity : targets and therapy 2019, 12: 75-83.

23. Kurinami N, Sugiyama S, Yoshida A, et al. Correlation of body muscle/fat ratio with insulin sensitivity using hyperinsulinemic-euglycemic clamp in treatment-naïve type 2 diabetes mellitus. Diabetes 
research and clinical practice 2016, 120: 65-72.

24. Hameed EK, AbdulQahar ZH. Visceral adiposity index in female with type 2 diabetic mellitus and its association with the glycemic control. Diabetes \& metabolic syndrome 2019, 13(2): 1241-1244.

25. Liu PJ, Ma F, Lou HP, Chen Y. Visceral Adiposity Index Is Associated with Pre-Diabetes and Type 2 Diabetes Mellitus in Chinese Adults Aged 20-50. Annals of nutrition \& metabolism 2016, 68(4): 235243.

26. Yatsuya H, Nihashi T, Li Y, et al. Independent association of liver fat accumulation with insulin resistance. Obesity research \& clinical practice 2014, 8(4): e350-355.

27. Guzmán G, Martínez V, Yara JD, et al. Glycemic Control and Hypoglycemia in Patients Treated with Insulin Pump Therapy: An Observational Study. J Diabetes Res 2020, 2020: 1581726.

28. Wainstein J, Metzger M, Boaz M, et al. Insulin pump therapy vs. multiple daily injections in obese Type 2 diabetic patients. Diabet Med 2005, 22(8): 1037-1046.

29. Guo Q, Zang P, Xu S, et al. Time in Range, as a Novel Metric of Glycemic Control, Is Reversely Associated with Presence of Diabetic Cardiovascular Autonomic Neuropathy Independent of HbA1c in Chinese Type 2 Diabetes. J Diabetes Res 2020, 2020: 5817074.

30. Lu J, Ma X, Shen Y, et al. Time in Range Is Associated with Carotid Intima-Media Thickness in Type 2 Diabetes. Diabetes technology \& therapeutics 2020, 22(2): 72-78.

31. Vigersky RA, McMahon C. The Relationship of Hemoglobin A1C to Time-in-Range in Patients with Diabetes. Diabetes technology \& therapeutics 2019, 21(2): 81-85.

32. Rodbard D. Glucose Time In Range, Time Above Range, and Time Below Range Depend on Mean or Median Glucose or HbA1c, Glucose Coefficient of Variation, and Shape of the Glucose Distribution. Diabetes technology \& therapeutics 2020, 22(7): 492-500.

33. Rodbard D. Glucose Variability: A Review of Clinical Applications and Research Developments. Diabetes technology \& therapeutics 2018, 20(S2): S25-s215.

34. Iwao T, Sakai K, Ando E. Relative contribution of insulin secretion and sensitivity at different stages of glucose tolerance: non-obese versus obese Japanese subjects. Internal medicine (Tokyo, Japan) 2014, 53(5): 383-390.

35. Kurinami N, Sugiyama S, Morita A, et al. Ratio of muscle mass to fat mass assessed by bioelectrical impedance analysis is significantly correlated with liver fat accumulation in patients with type 2 diabetes mellitus. Diabetes research and clinical practice 2018, 139: 122-130.

\section{Figures}


A

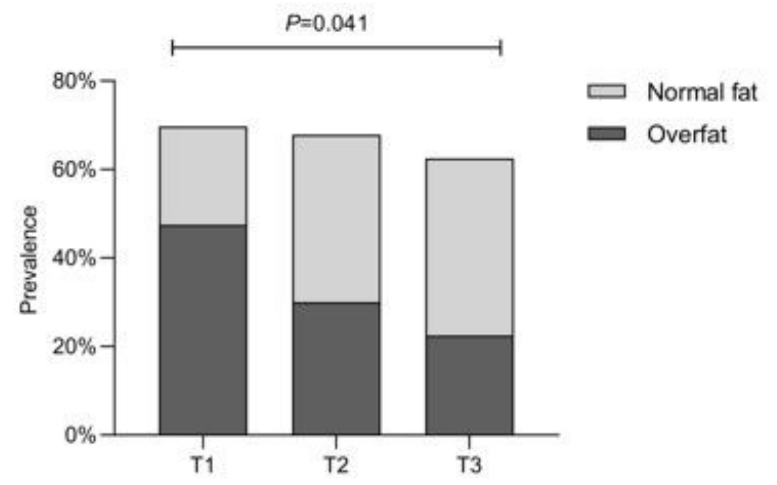

C

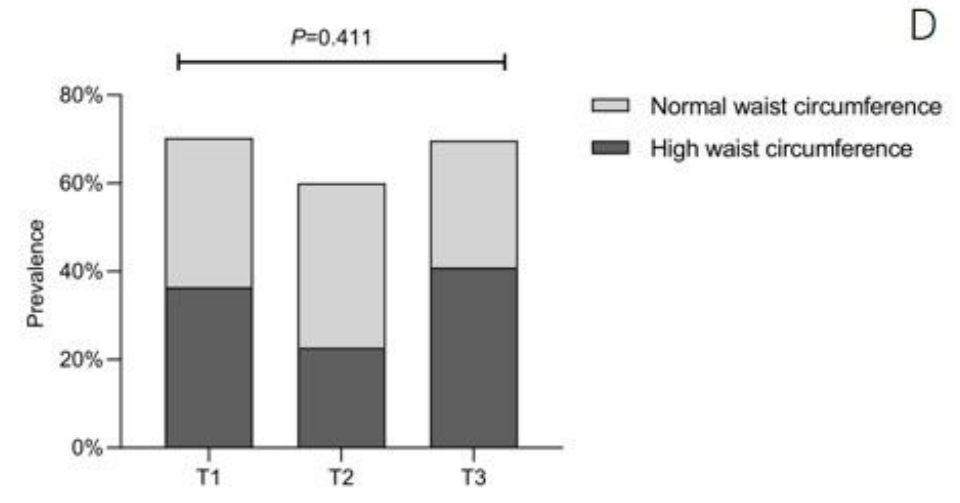

B

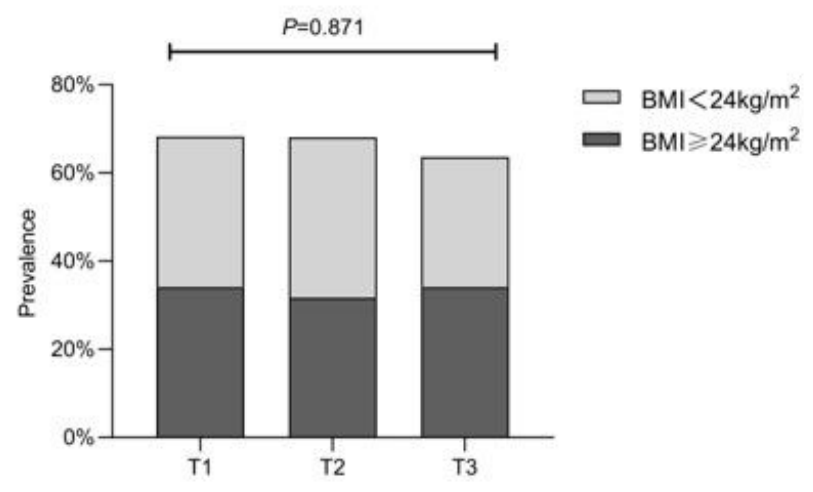

)

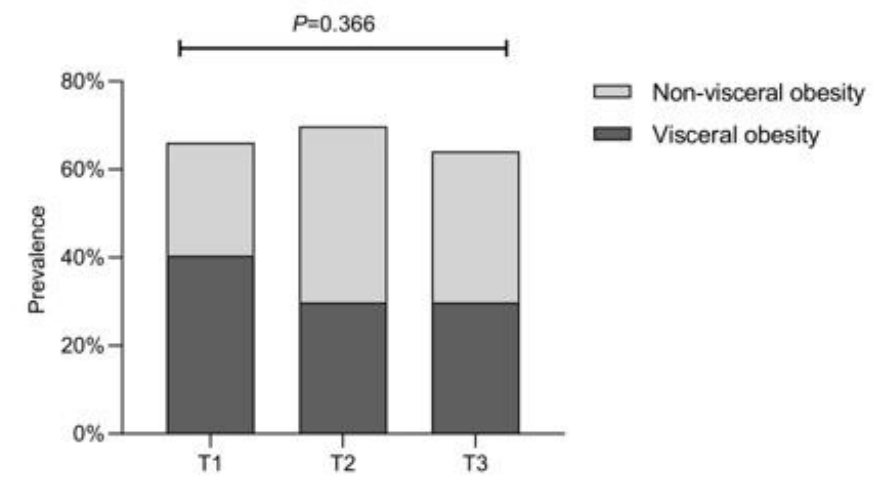

\section{Figure 1}

The proportion of participants stratified by body fat percentage, BMI, waist circumference or visceral fat area in different tertiles (T1-T3) of TIRa. Fig 1 (A): The participants were classed as normal fat and overfat. Overfat was defined as an amount of body fat of at least $25 \%$ of total body mass for men and at least $30 \%$ for women. Fig 1 (B): The participants were classed as underweight or normal weight (BMI $24 \mathrm{~kg} / \mathrm{m} 2)$ and overweight or obesity (BMI $\geq 24 \mathrm{~kg} / \mathrm{m} 2)$. Fig 1 (C): The participants were classed as normal waist circumference and high waist circumference. A waist circumference $>88 \mathrm{~cm}$ in women and $>102$ $\mathrm{cm}$ in men was classified as high waist circumference. Fig 1 (D): The participants were classed as visceral obesity and non-visceral obesity. The visceral obesity was defined as visceral fat area $\geq 100 \mathrm{~cm} 2$. a TIR T1 $\leq 43.75 \%, T 2: 43.76-57.29 \%, T 3: \geq 57.30 \%$. P value for the significant difference among the groups was determined by the chi-square test. 